Asian Spine Journal

Vol. 2, No. 2, pp 81 89, 2008

\title{
Axial Plane Lumbar Responses after Anterior Selective Thoracic Fusion for Main Thoracic Adolescent Idiopathic Scoliosis
}

\author{
$\mathrm{Ki}-\mathrm{Ho} \mathrm{Na}{ }^{*}$, Jürgen Harms ${ }^{\dagger}$, Kee-Yong $\mathrm{Ha}^{\dagger}$, Nam-Yong Choi ${ }^{*}$ \\ *Department of Orthopedic Surgery, St. Paul's Hospital, \\ The Catholic University of Korea College of Medicine, Seoul, Korea \\ ${ }^{\dagger}$ Department of Orthopedics and Traumatology, Center for Spinal Surgery, \\ Klinikum Karlsbad-Langensteinbach, Germany \\ ${ }^{\ddagger}$ Department of Orthopedic Surgery, Kang-Nam St. Mary's Hospital, \\ The Catholic University of Korea College of Medicine, Seoul, Korea
}

Study Design: A retrospective radiographic study.

Purpose: To evaluate the axial plane lumbar responses after anterior selective thoracic fusion (STF) in patients with main thoracic adolescent idiopathic scoliosis (MT-AIS).

Overview of Literature: Anterior scoliosis surgery induces more MT derotation through disc preparation than posterior surgery.

Methods: Twenty-eight MT-AIS patients treated with STF were evaluated after a minimum follow-up (FU) of 2 years. The MT and lumbar coronal angles, as well as the MT and lumbar rotational angles at the most rotated vertebrae were measured.

Results: At the last FU, the MT coronal correction and derotation rates were $65 \%$ and $41 \%$, respectively. The lumbar coronal correction rate was $61 \%$ but there was minimal lumbar derotation (2\%). Nine cases were decompensated (coronal balance $>10$ $\mathrm{mm}$ ). After surgery, the compensated and decompensated groups showed similar MT coronal and axial correction rates. During the FU, the MT and lumbar apecies rotated in the same direction $(r=0.443)$. In addition, significant MT derotation occurred in the decompensated group with increasing lumbar rotational correction loss. At the last FU, while the MT coronal correction was similar between the two groups, there was more MT derotation in the decompensated group. Furthermore, the MT rotational change was strongly associated with the coronal C7 plumb line position $(r=0.728)$.

Conclusions: After anterior STF in patients with MT-AIS, the final MT derotation is strongly associated with the coronal C7 plumb line position. During the FU, the excessive MT derotation in the decompensated group was attributed to excessive lumbar rotational correction loss.

Key Words: Adolescent idiopathic scoliosis, Selective thoracic fusion, Anterior spinal fusion, Derotation, Decompensation

\section{Introduction}

In the surgical treatment of main thoracic adolescent idiopathic scoliosis (MT-AIS), the mechanism of correction in anterior surgery is different from that in posterior surgery. While posterior scoliosis surgery depends on a forceful instrumental correction, anterior scoliosis surgery depends basically on the removal of discs. The most important structure related to torsional stiffness is the disc, particularly the

Received Nov 7, 2008; 1st revised Nov 18, 2008; accepted Nov 19, 2008

Corresponding author: Nam-Yong Choi, MD

Department of Orthopedic Surgery, St. Paul's Hospital, The Catholic University of Korea

620-56, Jeonnong-2-dong, Dongdaemoon-gu, Seoul, 130-709, Korea

Tel: +82-2-958-2159, 2288 Fax: +82-2-965-1456, E-mail: nychoimay@yahoo.co.kr 
anterior two-thirds of the annulus fibrosus. The removal of disc results in a $90 \%$ loss of torsional stiffness, while removal of the posterior structures including the facet joints results in a $30 \%$ loss of torsional stiffness ${ }^{1,2}$. Therefore, disc removal causes more derotation of the curve apex than removal of the posterior structures. Disc removal induces natural derotation before the instrumental correction maneuver, which is accompanied by a coupled coronal correction ${ }^{3}$. This derotation before instrumental correction enables shorter fusion from the upper to the lower end vertebrae of the MT curve.

Theoretically, anterior scoliosis surgery would cause less coronal decompensation than posterior scoliosis surgery because the coupled coronal-axial correction is a more balanced correction, and the shorter fusion provides more mobile transitional segments available for compensation. On the other hand, the forceful derotational maneuver in posterior scoliosis surgery corrects the coronal plane deformity without sufficient derotation.

After the introduction of the C-D instrument in 1980s, there have been many reports on the rotational correction following a so-called "derotational maneuver" in posterior scoliosis surgery. Thompson et al. ${ }^{4}$ reported a $34 \%$ thoracic derotation rate after selective thoracic correction surgery, and explained the derotation maneuver as the main cause of coronal decompensation. They suggested that surgical maneuver was a more important factor of coronal decompensation than the preoperative curve factors, such as the magnitude of the Cobb angle, curve flexibility, or the degree of apical rotation. Moreover, they believed that excessive derotation with longer fusion extending to the transitional area would transfer more rotational force to the lumbar curve, which would be unable to compensate for the thoracic correction because the lumbar rotational characteristics were changed. However, other reports showed that posterior instrumental correction induced no real derotation of the thoracic apex but en bloc relocation of the coronal curve to the sagittal plane ${ }^{5-8}$. Moreover, over-correction of the MT curve beyond the lumbar capacity was believed as the main cause of coronal decompensation ${ }^{9-11}$.

There are few reports on the lumbar response in the axial plane after anterior selective thoracic correction in adolescent idiopathic scoliosis. This study examined the effect of changes in the axial plane on the coronal decompensation as well as the lumbar responses in the axial plane after anterior selective thoracic fusion in patients with MT-AIS.

\section{Materials and Methods}

The radiographs of 28 patients with MT-AIS treated by anterior selective thoracic fusion were evaluated retrospectively. All the MT curves were right-sided. The images contained the curves with a Lenke's lumbar modifier A, B, and C. The following cases were excluded in order to minimize the selection bias: cases with proximal thoracic Cobb angles $>25^{\circ}$ on side-bending (Lenke type 2 curves); cases with lumbar modifier $\mathrm{A}$, in which the body center of lumbar apex did not cross the center sacral vertical line (CSVL) due to a severe L4 tilt; and cases where distal fusion exceeded more than one level distal to the lower end vertebra of the MT curves.

The patients underwent surgery between September 1994 and May 2004 in Klinikum Karlsbad-Langensteinbach, Germany. The mean age of the patients at surgery was 14 years and 8 months (range, 11.4 to 18.4 years). Of the 28 patients, 23 were female and 5 were male. The mean follow-up was 50.1 months (range, 25 to 116 months). A senior surgeon $(\mathrm{JH})$ performed the procedures using the standard surgical approach of one incision, double thoracotomy to obtain access to the entire MT vertebrae and occasionally up to the first lumbar vertebra ${ }^{12}$. The instrumentation used was a Moss Miami Spine System (Depuy Spine, Raynham, MA, USA) in 23 patients and a Moss Spine System (Depuy Spine) in 5 patients. All patients were operated once without revision.

The measurement was performed by one of the investigators $(\mathrm{KHN})$. Eight radiographs were taken for each patient; long cassette, posteroanterior and lateral standing radiographs without a brace at the preoperative period, immediately after surgery and at the last follow-up period, and long cassette preoperative supine active side-bending radiographs. In all cases, the radiographs taken immediately after surgery were checked 7 to 14 days after surgery.

In the coronal plane, the position of the $\mathrm{C} 7$ plumb line (C7 PL) was measured from the CSVL. It was described as (+) if it lay on the right side from the CSVL, and (-) if on the left side, and its change was described as (+) if it moved to the right side and (-) if it moved to the left side. The coronal Cobb angles were measured in MT and lumbar curves. The coronal balance was measured as the distance of the C7 PL from the CSVL.

In the axial plane, the rotational angles of the most rotated vertebrae at the MT and lumbar apecies were measured 
using Pedriolle's methods ${ }^{13}$. For more precise results, the rotational angle was measured in $2.5^{\circ}$ intervals instead of the conventional $5^{\circ}$ intervals. The rotational angles or angle changes were described as ( + ) if they were rotated to the right side and (-) if rotated to the left.

The thoracic kyphosis (T5-T12) and lumbar lordosis (T12-S1) were measured in the sagittal plane. According to a previous report of the changes in the segmental lordosis angle ${ }^{14}$, the unfused lumbar sagittal curve (LIV-S1) was divided by the lumbar apical vertebra (AV) into the proximal lumbar lordosis (the lower endplate of the LIV to that of the AV), and the distal lumbar lordosis (the lower endplate of the AV to the upper endplate of S1). The sagittal angles or angle changes were described as (+) if they were or became kyphotic, and (-) if lordotic.

Statistical analysis was carried out using SPSS ver 12.0 (SPSS Inc., Chicago, IL, USA). The change from preoperative to immediate postoperative values was described as a "surgical motion," and the change from immediate postoperative to the final values was described as a "follow-up motion". The rate of correction loss during the follow-up was based on the preoperative value (lost angle divided by the preoperative angle) in a similar manner to that of the correction rate. The motions of the coronal and axial parameters were expressed as the mean values, and the correlation between them was analyzed using Pearson's correlation and linear regression analysis. The effect of a change in the axial plane on the coronal decompensation was evaluated. The patients were subdivided into the compensated and decompensated groups according to the level of coronal decompensation (10 $\mathrm{mm}$ of the coronal balance). The coronal and axial plane motions were compared between the two groups.

\section{Results}

\section{Results in all patients}

The mean preoperative upper and lower lumbar end vertebra was 11.3 (11 means T11) and 16.0 (L4), respectively. The mean neutral vertebra was 11.8 . The mean lumbar apex was 14.5 between L2 (14) and L3 (15). The distal fusion level was 11.8, which was similar to the level of the neutral vertebra. An average of 6.8 segments were fused.

At the last follow-up, the lumbar Cobb angle was corrected $61 \%$ (mean, $35^{\circ}$ to $14^{\circ}$ ) while MT Cobb angle was cor- rected $65 \%$ (mean, $52^{\circ}$ to $18^{\circ}$ ) (Table 1 ). However, lumbar rotation was minimally corrected ( $2 \%$; mean, $-9.2^{\circ}$ to $-9.0^{\circ}$ ) while the MT rotation was corrected $41 \%$ (mean, $+24.6^{\circ}$ to $+14.2^{\circ}$ ). Finally, the coronal C7 PL was well maintained ($4.9 \mathrm{~mm}$ at the preoperative period, and $-6.0 \mathrm{~mm}$ at the last follow-up). Three cases had a $>20 \mathrm{~mm}$ coronal balance. From the definition of coronal decompensation being $>10$ mm of coronal balance, 19 cases were compensated and 9 cases were decompensated.

After selective thoracic fusion, most of the lumbar coronal correction was reported to occur above the lumbar apex $^{14,15}$. The explanation for this was that most of the coronal correction in the proximal lumbar area was possible because the proximal lumbar area had been mobilized in the sagittal plane ${ }^{14}$. The similar motion was observed in the axial plane. As the proximal lumbar area was mobilized in the sagittal plane, the lumbar apex was derotated, and the correlations were moderate ( $\mathrm{r}=0.411$ after surgery, $\mathrm{r}=0.445$ during follow-up) (Fig. 1).

It was previously reported that the same directional motion of the MT and lumbar apecies was observed in the coronal plane (coronal block motion) after surgery and during the follow-up at which time the coronal block motion was more evident during the follow-up than after surgery $(\mathrm{r}=0.467$ after surgery, $\mathrm{r}=0.808$ during the follow-up). Also, similar directional motion was observed in the axial plane, even though the correlation was weaker in the axial plane than the coronal plane. The correlation was more evident during the follow-up ( $\mathrm{r}=0.319$ after surgery, $\mathrm{r}=0.443$ during follow-up) (Fig. 2). This means that the lumbar apex rotated to the same side (left side) after surgery as the MT apex rotated to the left side. In addition, during the follow-up, the MT apex was rotated to the the same side (right side) as the lumbar apex rotated to the right side. It was called as axial block motion.

It was reported that the surgical motion of the coronal parameters were in the opposite direction to the follow-up motion. One explanation for this opposite directional motion was that the surgical motion was self-regulated during the follow-up. This self-regulation was also observed in the axial plane in a moderate to high correlation. As the MT apex was derotated more to the left side after surgery, it was rotated more to the opposite side (right side) during the follow-up $(\mathrm{r}=0.536)$. In addition, as the lumbar apex derotated more to the right side after surgery, it rotated more to the opposite side (left side) during the follow-up ( $\mathrm{r}=0.585$ ) (Fig. 3). 
Table 1. Coronal Cobb angles and axial rotational angles.

\begin{tabular}{|c|c|c|c|c|c|c|c|c|c|}
\hline & & \multicolumn{4}{|c|}{ Main thoracic $\left({ }^{\circ}\right)$} & \multicolumn{4}{|c|}{ Lumbar $\left({ }^{\circ}\right)$} \\
\hline & & SB & Pre & IPO & Last & SB & Pre & IPO & Last \\
\hline \multicolumn{10}{|c|}{ All patients $(\mathrm{n}=28)$} \\
\hline Coronal & mean & 26.2 & 52.0 & 14.0 & 18.1 & 2.9 & 35.0 & 14.2 & 13.6 \\
\hline Cobb & minimum & 7 & 38 & -1 & 5 & -20 & 18 & 4 & 3 \\
\hline \multirow[t]{2}{*}{ angle } & maximum & 49 & 72 & 30 & 32 & 25 & 49 & 28 & 31 \\
\hline & $\mathrm{SD}$ & 10.7 & 9.2 & 8.4 & 7.4 & 10.6 & 7.0 & 6.3 & 8.4 \\
\hline \multirow{4}{*}{$\begin{array}{l}\text { Axial } \\
\text { rotational } \\
\text { angle }\end{array}$} & mean & & 24.8 & 14.6 & 14.6 & & -9.2 & -7.9 & -9.0 \\
\hline & minimum & & 5 & 2.5 & -5 & & -2.5 & -2.5 & -2.5 \\
\hline & maximum & & 37.5 & 32.5 & 32.5 & & -20 & -17.5 & -22.5 \\
\hline & $\mathrm{SD}$ & & 8.8 & 8.6 & 8.8 & & 5.0 & 4.2 & 5.8 \\
\hline \multicolumn{10}{|c|}{ Compensated group (C-group) $(n=19)$} \\
\hline \multirow{4}{*}{$\begin{array}{l}\text { Coronal } \\
\text { Cobb } \\
\text { angle }\end{array}$} & mean & 25.2 & 51.3 & 14.2 & 19.2 & 2.0 & 33.8 & 13.6 & 13.3 \\
\hline & minimum & 7 & 38 & -1 & 6 & -12 & 18 & 4 & 3 \\
\hline & maximum & 40 & 68 & 30 & 32 & 23 & 45 & 23 & 31 \\
\hline & $\mathrm{SD}$ & 9.3 & 8.4 & 9.4 & 7.5 & 9.2 & 6.2 & 5.8 & 7.9 \\
\hline \multirow{4}{*}{$\begin{array}{l}\text { Axial } \\
\text { rotational } \\
\text { angle }\end{array}$} & mean & & 24.6 & 14.2 & 16.2 & & -8.8 & -7.6 & -8.3 \\
\hline & minimum & & 5 & 5 & 5 & & -2.5 & -2.5 & -2.5 \\
\hline & maximum & & 35 & 32.5 & 32.5 & & -15 & -17.5 & -17.5 \\
\hline & SD & & 8.6 & 7.9 & 8.8 & & 4.7 & 4.2 & 4.9 \\
\hline \multicolumn{10}{|c|}{ Decompensated group (D-group) $(n=9)$} \\
\hline Coronal & mean & 28.2 & 53.4 & 13.4 & 15.8 & 4.9 & 37.4 & 15.4 & 14.4 \\
\hline Cobb & minimum & 16 & 42 & 6 & 5 & -20 & 26 & 6 & 4 \\
\hline angle & maximum & 49 & 72 & 26 & 27 & 25 & 49 & 28 & 31 \\
\hline \multirow{5}{*}{$\begin{array}{l}\text { Axial } \\
\text { rotational } \\
\text { angle }\end{array}$} & $\mathrm{SD}$ & 13.5 & 11.0 & 6.2 & 6.9 & 13.5 & 8.4 & 7.6 & 10.0 \\
\hline & mean & & 25.3 & 15.6 & 11.4 & & -10.0 & -8.3 & -11.0 \\
\hline & minimum & & 7.5 & 2.5 & -5 & & -2.5 & -2.5 & -2.5 \\
\hline & maximum & & 37.5 & 27.5 & 20 & & -20 & -15 & -22.5 \\
\hline & $\mathrm{SD}$ & & 9.6 & 10.4 & 8.4 & & 5.6 & 4.3 & 7.6 \\
\hline
\end{tabular}

SB: sibe-bending, Pre: preoperative, IPO: immediate postoperative, Last: last follow-up, Decompensation was defined as more than $10 \mathrm{~mm}$ deviation of the $\mathrm{C} 7$ plumb line from the center sacral vertical line at the last follow-up, SD: standard deviation.
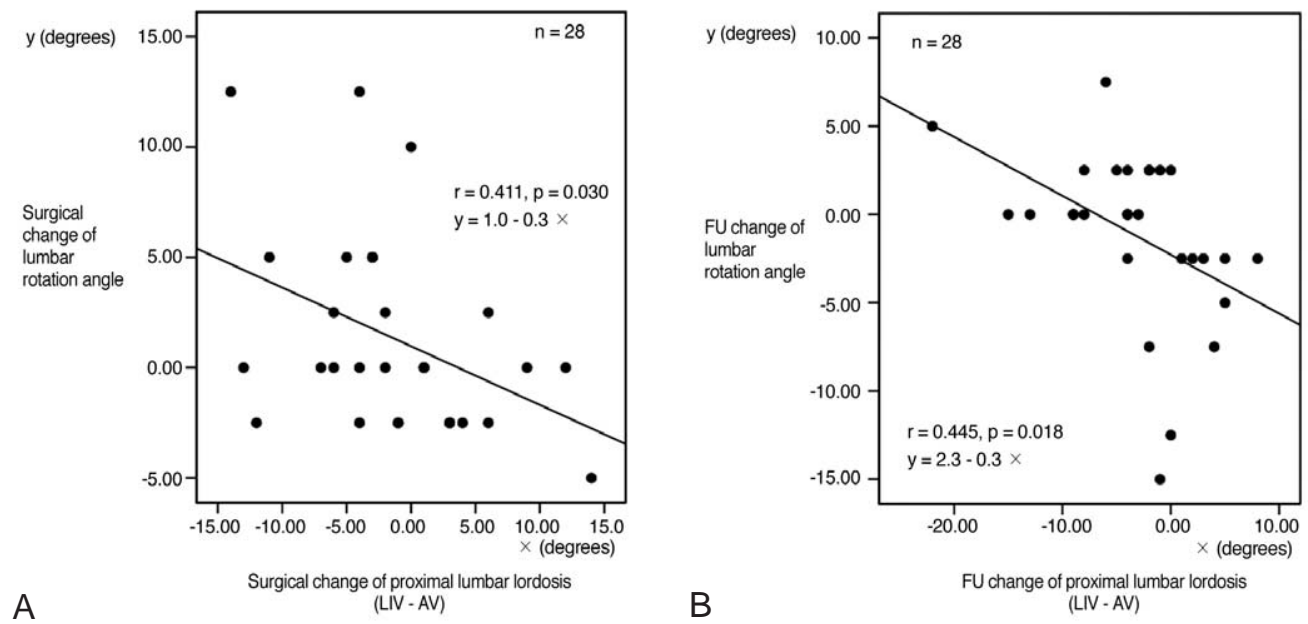

Fig. 1. Sagittal plane change in the proximal lumbar area (lowest instrumented vertebra - apical vertebra) was moderately correlated with the rotational angle change in the lumbar apex immediately after surgery (A), and during follow-up (B). 


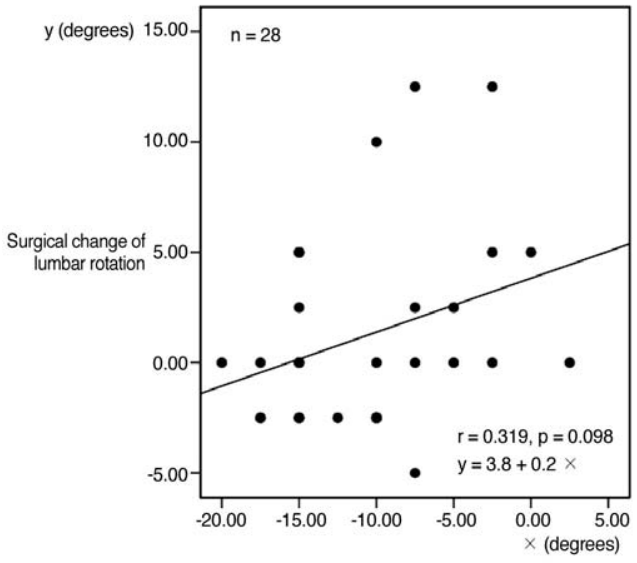

A

Surgical change of MT rotation

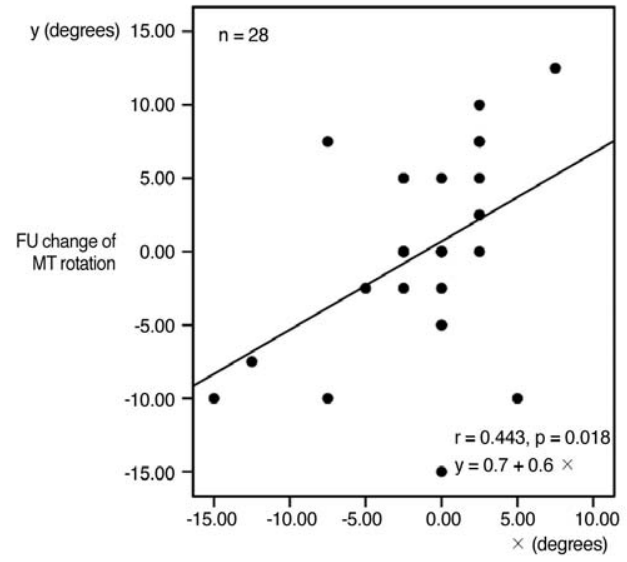

B

FU change of lumbar rotation

Fig. 2. Axial block motion. Main thoracic apex and lumbar apex tend to rotate to the same side. Axial block motion immediately after surgery (A), and during the follow-up (B).
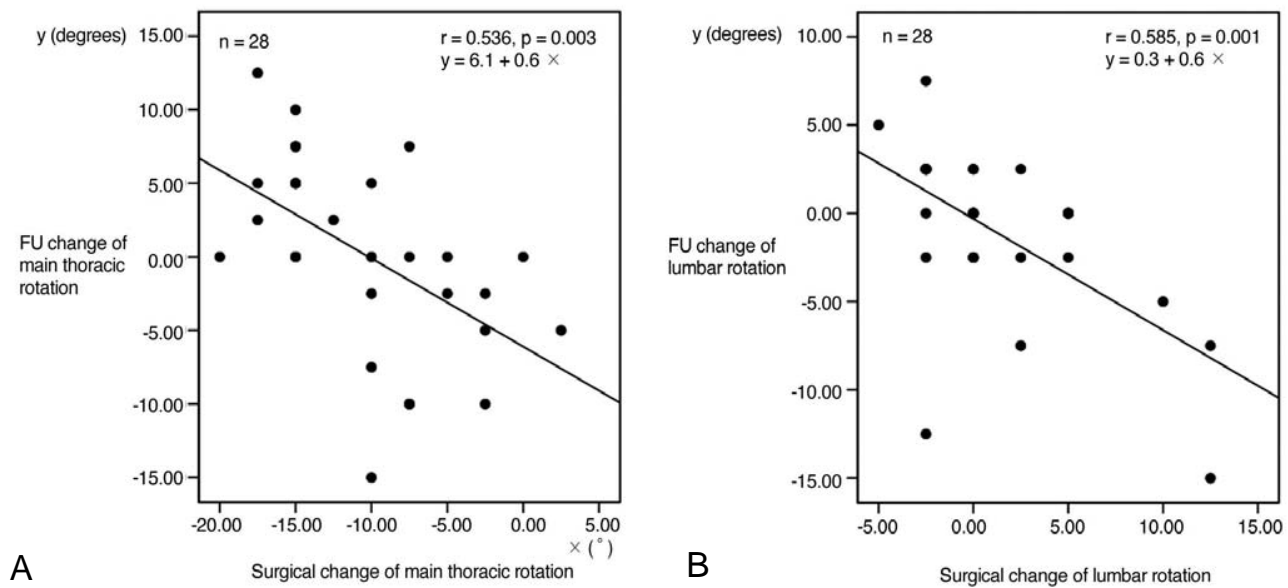

Fig. 3. Self-regulation in the axial plane. The follow-up motion tends to be in the opposite direction to the surgical motion, which suggests that surgical motion is regulated during the follow-up. Self-regulation of the MT apical rotational angle (A), and of lumbar apical one (B).

\section{Comparisons of the compensated and the decom- pensated groups}

According to the definition of coronal decompensation being $>10 \mathrm{~mm}$ of coronal balance, 19 cases were compensated, and 9 cases were decompensated. There was no significant difference between the two groups in terms of age, gender, magnitude of the coronal Cobb angle, coronal flexibility and rotational angle ( $p>0.05)$.

Immediately after surgery, the compensated and decompensated groups showed similar MT coronal correction rates $(72.3 \%$ vs. $74.9 \%$, respectively) ( $\mathrm{p}=0.656$, MannWhitney test), MT rotational correction rates $(42.3 \%$ vs. $38.3 \%$, respectively) $(\mathrm{p}=0.927)$, lumbar coronal correction rates $(59.8 \%$ vs. $58.8 \%$, respectively) $(\mathrm{p}=0.911)$, and lum- bar rotational correction rates (13.6\% vs. $10.0 \%$, respectively) $(\mathrm{p}=0.537)$ (Table 2).

However, during the follow-up, the MT coronal correction loss was similar in the compensated vs. decompensated groups $(9.7 \%$ correction loss vs. $4.5 \%$ correction loss, respectively) $(\mathrm{p}=0.108)$ but there was a difference in MT rotational correction loss in the two groups $(8.2 \%$ correction loss vs. $16.6 \%$ improved correction, respectively) $(\mathrm{p}=0.041)$. The lumbar coronal Cobb angle was corrected continuously in the two groups ( $0.9 \%$ improved vs. $2.7 \%$ improved, respectively) $(\mathrm{p}=0.704)$. However, the lumbar rotational correction was lost during the follow-up in both groups, and there was more loss in the decompensated group $\left(0.7^{\circ}\right.$ correction loss vs. $2.7^{\circ}$ correction loss, respectively), even though the difference was not significant 
Table 2. Coronal and axial correction rates, and coronal C7 plumb line position

\begin{tabular}{|c|c|c|c|c|c|c|c|c|}
\hline & & \multicolumn{2}{|c|}{$\begin{array}{c}\text { Main thoracic } \\
\text { correction rate }(\%)\end{array}$} & \multicolumn{2}{|c|}{$\begin{array}{c}\text { Lumbar } \\
\text { correction rate }(\%)\end{array}$} & \multicolumn{3}{|c|}{$\begin{array}{l}\text { Coronal C7 PL } \\
\text { position }(\mathrm{mm})\end{array}$} \\
\hline & & IPO & Last & IPO & Last & Pre & IPO & Last \\
\hline All Patients & Cobbs & 73.1 & 65.2 & 59.4 & 61.1 & -4.9 & -1.6 & -6.0 \\
\hline$(\mathrm{n}=28)$ & Rotation & 41.1 & 41.1 & 14.1 & 2.2 & & & \\
\hline C-group & Cobbs & 72.3 & 62.6 & 59.8 & 60.7 & -5.1 & 1.1 & -1.6 \\
\hline$(n=19)$ & Rotation & 42.3 & $34.1 *$ & 13.6 & 5.7 & & & \\
\hline D-group & Cobbs & 74.9 & 70.4 & 58.8 & 61.5 & -4.4 & -7.4 & -15.4 \\
\hline$(n=9)$ & Rotation & 38.3 & $54.9 *$ & 17.0 & -6.0 & & & \\
\hline
\end{tabular}

* Significant differences by Mann-Whitney U test, C-group, compensated group, D-group, decompensated group, Cobbs, Cobb angles, rotation, rotational angles, Coronal C7 PL position, position of C7 plumb line from the center sacral vertical line, (+) means right position, (-) means left position, IPO: immediate postoperative, Last: last follow-up, Pre: preoperative.
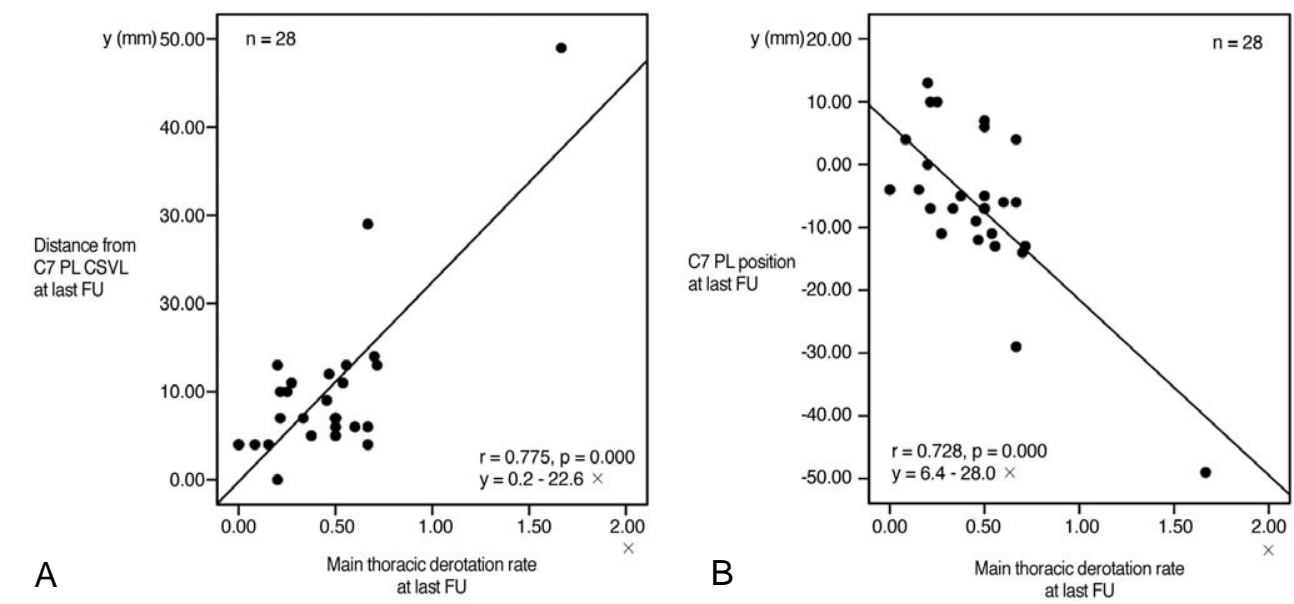

Fig. 4. The distance of the $\mathrm{C} 7$ plumb line from center sacral vertical line at the last follow-up is associated with the main thoracic (MT) apical derotation rate at the last follow-up (A). At last follow-up, the final C7 PL lies more on the left side with increasing correction of MT rotation (B). CSVL: center sacral vertical line, PL: plumb line.

$(\mathrm{p}=0.851)$. In the decompensated group, the final lumbar rotational angle $\left(-11.0^{\circ}\right)$ was larger than the preoperative one $\left(-10.0^{\circ}\right)$.

After surgery, the coronal balance (coronal C7 PL position) was improved in the compensated group $(-5.1 \mathrm{~mm}$ to $+1.1 \mathrm{~mm}$ ) but aggravated in the decompensated group (-4.4 $\mathrm{mm}$ to $-7.4 \mathrm{~mm}$ ). During the follow-up, the coronal C7 PL position was well maintained in the compensated group (+ $1.1 \mathrm{~mm}$ to $-1.6 \mathrm{~mm})$ but more aggravated in the decompensated group (-7.4 mm to $-15.4 \mathrm{~mm}$ ).

At the last follow-up, while both groups showed a similar final MT coronal correction rate $(62.6 \%$ vs. $70.4 \%$, respectively) ( $\mathrm{p}=0.113)$, the MT rotational correction rate was significantly higher in the decompensated group $(34.1 \%$ vs. $54.9 \%$, respectively) $(\mathrm{p}=0.022)$. In addition, there was a strong correlation between the final MT rotational correction rate and final coronal C7 PL deviation (distance of C7
PL from the CSVL) $(r=0.775)$. Precisely speaking, at last follow-up, there was more movement of the final C7 PL to the left side with increasing level of MT derotation (r=0.728) (Fig. 4B).

\section{Discussion}

The rotational angle was measured from the standing radiographs using the Pedriolle's method. CT-guided measurements of the rotational angle is known to be the most correct method of measurement, but it determines the angle in the supine position and a follow-up CT is generally unavailable in most cases due to the higher cost. However, Yazici et al. ${ }^{16}$ reported that the rotational angle measured using Perdriolle's method in the supine plain radiograph was similar to the rotational angle measured by $\mathrm{CT}$ in the 
supine position.

Most reports describing the axial plane response examined cases treated with posterior scoliosis surgery, and most of those dealt with the preoperative and final values. It is believed that the material treated by anterior scoliosis surgery would be a better model of the lumbar responses after selective thoracic correction than that treated by posterior scoliosis surgery. This is because anterior scoliosis surgery depends basically on disc removal rather than vigorous instrumental correction, which results in a more balanced three-dimensional correction.

This study examined the values immediately after surgery. In addition, it is postulated that the change from the preoperative to immediate postoperative values was the result of surgical motion, and the change from the immediate postoperative to the final values is a follow-up motion.

At the last follow-up, the MT derotation rate was $41.1 \%$, which was similar to the values reported in the literatures ${ }^{17,18}$. As mentioned above, there was minimal MT derotation when corrected by posterior scoliosis surgery without the removal of discs. In this study, the MT derotation was $41.1 \%$ after a multi-level discectomy. The operator removed the discs at all levels included in the fusion in order to increase the correction rate. While the MT coronal Cobb angle was corrected $65.2 \%$, the MT rotational angle was corrected $41.1 \%$. On the other hand, while lumbar coronal Cobb angle was corrected $61.1 \%$, the lumbar rotational angle was corrected by only $2.2 \%$. There was also minimal lumbar derotation without the removal of discs.

Changes similar to the coronal plane changes ${ }^{14}$ were observed in the axial plane. The lumbar apex derotated more in the axial plane with increasing mobilization of the proximal lumbar area in the sagittal plane (Fig. 1). The MT and lumbar apecies rotated to the same direction (axial block motion), which was more evident during the followup than after surgery (Fig. 2). The surgical motion of the MT or lumbar rotational angle was regulated during the follow-up (Fig. 3). However, the correlations in the axial plane were poorer than that in the coronal plane $\mathrm{e}^{14}$. This was attributed to an axial plane deformity being more structural than the coronal plane deformity. According to the concept of biplanar asymmetry ${ }^{19,20}$, rotatory momentum develops in the axial plane as the MT area is mobilized in the sagittal plane. In addition, the increased axial momentum causes migration of the MT apex to the lateral side in the coronal plane. Therefore, the scoliotic image in the coronal plane is only a secondary phenomenon, the primary event being rotation.
The side bending force releases the coronal Cobb angle but not the axial plane angle ${ }^{21,22}$. It is believed that the axial plane deformity is the primary event, and becomes more structural than the coronal plane deformity.

In general, the rotational angle will improve if the coronal angle improves and vice versa. This is known as coronalaxial coupled motion. After surgery, coronal-axial coupled motion occurred in the MT and lumbar curve. However, coupled motion did not occur during the follow-up. In the MT curve, while the MT coronal correction was lost during the follow-up, the MT rotational correction was also lost in compensated group (coupled motion), however the MT rotational correction was improved in decompensated group (de-coupled motion). On the other hand, in the lumbar curve, while spontaneous lumbar coronal correction occurred during the follow-up, the lumbar rotational correction was also lost in both groups (de-coupled motion). The only way of explaining this phenomenon is through two concepts; the rigidity of the axial plane deformity, and the axial block motion between the MT and lumbar curves.

At first, the axial plane is most structural among the three planes. Posterior scoliosis surgery without disc removal could not correct the axial plane deformity well. Without disc removal, the rotational deformity is not easily corrected, regardless of whether it is MT or lumbar curve. In addition, although the lumbar apex derotated after surgery, the derotation was lost during the follow-up. This means that the lumbar derotation had returned to its preoperative state during the follow-up.

The second rationale explaining the above mentioned coronal-axial de-coupled motion is axial block motion. After selective thoracic fusion surgery, the MT correction is the primary event that occurs by surgical correction, and the lumbar curve responds to compensate after a MT correction. However, during the follow-up, the MT area is only a fused mass, and the MT curve should follow the spontaneous lumbar correction continuing during the follow-up. As mentioned above, in all patients, there was a moderate tendency of the MT and lumbar curves to rotate in the same direction as a unit, which was more evident during the follow-up (r=0.443) (Fig 2). Focusing on this axial block motion, the axial plane motion was analyzed comparing both groups. During the follow-up, there was significantly higher derotation of the MT apex in the decompensated group than in the compensated group $\left(+2.0^{\circ}\right.$ vs. $-4.2^{\circ}$, respectively) (8.2\% correction loss vs. $16.6 \%$ improved correction, respectively) $(\mathrm{p}=0.041)$. Meanwhile, there was 
more loss of the lumbar rotational correction in the decompensated group $\left(-0.7^{\circ}\right.$ vs. $-2.7^{\circ}$, respectively) but the difference was not statistically significant $(\mathrm{p}=0.851)$. It is believed that axial block motion is responsible for the excessive derotation of the MT apex during the follow-up. Following the excessive correction loss of lumbar apical rotation (rotation to the left side), the passive MT fused mass was rotated into the same direction (rotation to the left side). This resulted in excessive left side rotation of the MT apex.

A comparison of the MT coronal correction rates between the compensated and decompensated groups showed no significant difference after surgery and during the follow-up. However, there was a significant difference in the MT rotational correction rate during the follow-up. At the last follow-up, while the MT coronal correction was similar in the two groups (63\% vs. $70 \%$, respectively) $(\mathrm{p}=0.113)$, there was more MT derotation in the decompensated group (34\% vs. $55 \%$, respectively) $(\mathrm{p}=0.041)$. In addition, there was a strong correlation between the final MT rotational correction rate and the final coronal C7 PL deviation ( $r=0.775)$ (Fig. 4A). Linear regression analysis revealed the following equation.

$$
\mathrm{y}=-0.2+22.6 x
$$

where $\mathrm{y}=$ distance of C7 PL from CSVL $(\mathrm{mm})$, and $\mathrm{x}=$ the final MT rotational correction rate

According to the equation, a final MT rotational correction rate $>45 \%$ induces $>10 \mathrm{~mm}$ movement of the $\mathrm{C} 7 \mathrm{PL}$ position away from the CSVL. The equation can explains the final MT rotational correction rate of $44.1 \%$ in all patients; $34.1 \%$ in the compensated group, and $54.9 \%$ in the decompensated group. Among the 9 patients in decompensated group, 7 showed a final MT rotational correction rate $>45 \%$. In addition, the coronal C7 PL position moved more to the left side with increasing final MT rotational correction (rotated to the left side), (Fig. 4B). This showed the fact that decompensation is the excessive left translation of the C7 PL in the coronal plane ${ }^{9-11}$. Overall, during the followup, more rigid lumbar rotational deformity in the decompensated group induced more rotational correction loss (rotation to left side), which induced more MT rotation to the left side. There was more coronal C7 PL deviation to the left side with increasing level of left rotation of the MT apex, which is more prone to coronal decompensation.

Although there was greater loss of lumbar rotational correction in the decompensated group than in the compensated group during the follow-up, the difference was not statis- tically significant. In this study, there was minimal change in lumbar rotational angle after surgery and during the follow-up. Because an axial plane deformity is more structural than a coronal plane deformity, the axial plane parameter showed more variation than the coronal plane parameter. While the ratio of one standard deviation to the preoperative value was $18 \%$ in the MT coronal angle, it was $35 \%$ in the MT rotational angle. Moreover, one standard deviation was $20 \%$ in the lumbar coronal angle but $54 \%$ in the lumbar rotational angle (Table 1). Therefore, gaining statistical significance is difficult in the axial plane, particularly in the lumbar area, because disc removal had not been performed in the lumbar curve, and there were variations in structural characteristics of the lumbar rotation. It is believed that a study with more cases would show statistical difference.

\section{Conclusions}

After anterior selective thoracic fusion in patients with MT-AIS, there was a strong correlation between the final MT derotation rate and the final C7 plumb line position, i.e., coronal decompensation. At the last follow-up, the coronal C7 PL position moved more toward the left side with increasing correction of MT rotation. During the follow-up period, excessive derotation of the MT apex and excessive correction loss of the lumbar apical rotation occurred in the decompensated group, and it is believed that excessive derotation of the MT apex was caused by excessive correction loss of the lumbar apical rotation.

\section{REFERENCES}

1. Markolf KL: Deformation of the thoracolumbar intervertebral joints in response to external loads: a biomechanical study using autopsy material. J Bone Joint Surg Am 1972; 54: 511-533.

2. Haher TR, Felmy W, Baruch H, et al: The contribution of the three columns of the spine to rotational stability. A biomechanical model. Spine 1989; 14: 663-669.

3. Dickson RA, Lawton JO, Archer IA, Butt WP: The pathogenesis of idiopathic scoliosis. Biplanar spinal asymmetry. J Bone Joint Surg Br 1984; 66: 8-15.

4. Thompson JP, Transfeldt EE, Bradford DS, Ogilvie JW, Boachie-Adjei O: Decompensation after CotrelDubousset instrumentation of idiopathic scoliosis. Spine 
1990; 15: 927-931.

5. Labelle H, Dansereau J, Bellefleur C, et al: Comparison between preoperative and postoperative three-dimensional reconstructions of idiopathic scoliosis with the CotrelDubousset procedure. Spine 1995; 20: 2487-2492.

6. Wood KB, Transfeldt EE, Ogilvie JW, Schendel MJ, Bradford DS: Rotational changes of the vertebral-pelvic axis following Cotrel-Dubousset instrumentation. Spine 1991; 16: S404-408.

7. Gray JM, Smith BW, Ashley RK, LaGrone MO, Mall J: Derotational analysis of Cotrel-Dubousset instrumentation in idiopathic scoliosis. Spine 1991; 16: S391-393.

8. Krismer M, Bauer R, Sterzinger W: Scoliosis correction by Cotrel-Dubousset instrumentation. The effect of derotation and three dimensional correction. Spine 1992; 17: S263-269.

9. Bridwell KH, McAllister JW, Betz RR, Huss G, Clancy M, Schoenecker PL: Coronal decompensation produced by Cotrel-Dubousset "derotation" maneuver for idiopathic right thoracic scoliosis. Spine 1991; 16: 769-777.

10. Moore MR, Baynham GC, Brown CW, Donaldson DH,

Odom JA Jr: Analysis of factors related to truncal decompensation following Cotrel-Dubousset instrumentation. J Spinal Disord 1991; 4: 188-192.

11. Arlet V, Marchesi D, Papin P, Aebi M: Decompensation following scoliosis surgery: treatment by decreasing the correction of the main thoracic curve or "letting the spine go”. Eur Spine J 2000; 9: 156-160.

12. Lenke LG, Betz RR, Harms J: Modern anterior scoliosi surgery 1st ed. St. Louis, Quality Medical Publishing Inc: 2004; 225-231.

13. Perdriolle R, Vidal J: Morphology of scoliosis: three- dimensional evolution. Orthopedics 1987; 10: 909-915.

14. Na KH, Harms J, Ha KY, Choi NY: Sagittal plane lumbar responses after anterior selective thoracic fusion for main thoracic adolescent idiopathic scoliosis. Asian Spine J 2007; 1: 80-87.

15. Mason DE, Carango P: Spinal decompensation in CotrelDubousset instrumentation. Spine 1991; 8: S394-403.

16. Yazici M, Acaroglu ER, Alanay A, Deviren V, Cila A, Surat A: Measurement of vertebral rotation in standing versus supine position in adolescent idiopathic scoliosis. $\mathrm{J}$ Pediatr Orthop 2001; 21: 252-256.

17. Schulte TL, Liljenqvist U, Hierholzer E, et al: Spontaneous correction and derotation of secondary curves after selective anterior fusion of idiopathic scoliosis. Spine 2006; 31: 315-321.

18. Muschik MT, Kimmich H, Demmel T: Comparison of anterior and posterior double-rod instrumentation for thoracic idiopathic scoliosis: results of 141 patients. Eur Spine J 2006; 15: 1128-1138.

19. Dickson RA, Lawton JO, Archer IA, Butt WP: The pathogenesis of idiopathic scoliosis. Biplanar spinal asymmetry. J Bone Joint Surg Br 1984; 66: 8-15.

20. Castelein RM, van Dieen JH, Smit TH: The role of dorsal shear forces in the pathogenesis of adolescent idiopathic scoliosis--a hypothesis. Med Hypotheses 2005; 65: 501508.

21. Matsumoto T, Kitahara H, Minami S, et al: Flexibility in the scoliotic spine: three-dimensional analysis. J Spinal Disord 1997; 10: 125-131.

22. Beuerlein MJ, Raso VJ, Hill DL, Moreau MJ, Mahood JK: Changes in alignment of the scoliotic spine in response to lateral bending. Spine 2003; 28: 693-698. 